P051

ACCURACY OF SEXUALLY TRANSMITTED INFECTIONS TESTING ON SELF-COLLECTED VAGINAL SAMPLES VERSUS CERVICAL SAMPLES

${ }^{1}$ Clementina Cocuzza*, ${ }^{1}$ Marianna Martinelli, ${ }^{1}$ Arianna Gallone, ${ }^{1}$ Rosario Musumeci, ${ }^{1}$ Benedetta Montanini, ${ }^{2}$ Federica Sina, ${ }^{2}$ Stefania Chiara, ${ }^{1}$ Robert Fruscio, ${ }^{1}$ Fabio Landoni. ${ }^{1}$ Department of Medicine and Surgery, University of Milano-Bicocca, Monza, Italy; ${ }^{2}$ San Gerardo Hospital, Gynaecology Division, Monza, Italy

\subsection{6/sextrans-2019-sti.256}

Background Self-sampling has been shown to be a non-invasive and cost-effective method for the diagnosis and screening of sexually transmitted infections (STI). This study aims to evaluate the accuracy of detection of HPV and other STI on self-collected vaginal samples as compared to clinician-collected cervical samples in women with a recent diagnosis of cervical dysplasia.

Methods Self-collected vaginal (VS) and physician-administered cervical samples (CS) were collected from 130 women attending the Colposcopy Clinic, San Gerardo Hospital, Monza, Italy with a diagnosis of cervical dysplasia. VS and CS were collected using FLOQSwabs and L-Shaped FLOQSwab (Copan) respectively and transported to the Microbiology Laboratory of the University of Milano-Bicocca. Samples' nucleic acid extraction was performed using NucliSENS ${ }^{\circledR}$ easyMAG (bioMérieux). HPV and STIs detection was evaluated using Anyplex II HPV28 and STI-7 (Seegene), respectively. Sample cellularity adequacy through human CCR5 gene assessment was performed using an 'in house' Real-time PCR assay.

Results Demonstrated a very good overall concordance for HPV and STI detection on self and clinician-collected samples (gold standard). Very good agreement for the detection of one or more HPV types was demonstrated (Kappa $=0.915$ ) with HPV positivity rates of $75 \%$ and $72 \%$ for VS and CS respectively. Similarly very good agreement was demonstrated for the detection of one or more of the 7 STIs understudy (Kappa $=0.899)$. Overall a higher positivity for STIs was found in VS (48\%) compared to CS (43\%), with Ureaplasma parvum being most frequently detected. Adequate sample cellularity was demonstrated for all samples types; mean values of $2.07 \mathrm{E}+06$ and $3.16 \mathrm{E}+06$ cells/sample for VS and CS respectively.

Conclusion Self-collected samples showed a high degree of concordance with CS for both HPV and STIs detection with comparable sample adequacy. These results are promising for the introduction of self-collected samples in sexually transmitted and cervical cancer screening programs.

Disclosure No significant relationships.

\section{P052 HIV CASE FINDING AND LINKAGE TO CARE IN ELEME LOCAL GOVERNMENT AREA, RIVERS STATE}

${ }^{1}$ Atochi Emenike*, ${ }^{2}$ Ochedomi Ekele, ${ }^{3}$ Golden Owhonda. 'Pathfinder International, Monitoring, Evaluation and Learning, Port Harcourt, Nigeria; ${ }^{2}$ Family Health International, FHI360, Monitoring and Evaluation, Port Harcourt, Nigeria; ${ }^{3}$ Rivers State Ministry of Health, Public Health, Port Harcourt, Nigeria

10.1136/sextrans-2019-sti.257

Background A combination of effective and efficient approaches are necessary for scaling up of HIV case-identification, particularly in resource-limited settings. To increase access and coverage, communities need to be linked to facilities via community-based interventions that seek to promote health seeking behavior. This study is a Comparative Analysis of Facility Optimization and Community Based HIV Intervention and compares the effect of community HIV testing services (HTS) to HTS optimization at the facility-level.

Methods This is a pre-and post-intervention study conducted in Eleme, one of the priority LGA supported by the USAID funded Strengthening Integrated Delivery of HIV/AIDS Services (SIDHAS) in Rivers State. The pre-intervention phase (PIP1) covers the period November 2015 - October 2016 while the post intervention phase is from November 2016 Oct 2017. PIP1 involved community entry/mobilization, HIV screening in general population, referrals and linkage to care and treatment services from the community to the facility, while the PIP2 focused on optimization of HIV testing services within the facilities through multipoint/Provider Initiated Testing and Counselling[PITC], targeted testing in the communities, Sexual Network and Genealogy Testing and referrals by escort to Service Delivery Points. We reviewed HTS and ART commencement data to compare differences in positivity yield and linkage between both phases.

Results The PIP1 had 107,813 individuals counselled, tested and received result, 1,406 tested HIV Positive and 964 linked to ART while the PIP2 had 24,078 individuals tested, 614 HIV positive and 610 linked to ART. Findings show increase in positivity yield from $1 \%$ to $3 \%$ and linkage from $87 \%$ to 99\% in PIP1 and PIP2 respectively.

Conclusion Although community outreaches create awareness, a targeted approach to HTS including sexual network/genealogy testing may be a more efficient approach. In addition, PITC in health facilities yields a higher positivity and linkage rates, maximizes use of testing resources by focusing on higher risk populations.

Disclosure No significant relationships.

\section{P053 DETECTION OF SEXUALLY TRANSMITTED PATHOGENS FROM SEDIMENT OF FIRST-VOID URINE IN PATIENTS FROM GREATER ZAGREB AREA}

${ }^{1} J a s m i n a$ Vranes*, ${ }^{2}$ Jakov Jurcevic, ${ }^{3}$ Jasna Knezevic, ${ }^{3}$ Vladimira Ticic, ${ }^{3}$ Suncanica LjubinSternak. 'Institute of Public Health 'Dr. Andrija Stampar', Department of Clinical Microbiology, Zagreb, Croatia; ${ }^{2}$ Zagreb University Medical School, Zagreb, Croatia; ${ }^{3}$ Institute of Public Health 'Dr. Andrija Stampar', Zagreb, Croatia

\subsection{6/sextrans-2019-sti.258}

Background First-void urine (FVU) is the preferred specimen for the diagnosis of sexually transmitted (ST) infections in men. The use of the invasive and painful urethral swabs is a major barrier to screening and a key factor in male nonattendance at genitourinary medicine clinics. The aim of this study was detection of ST pathogens from sediment of FVU of 822 men, collected instead of urethral swabs.

Methods All samples were stained, cultivated and used in multiplex polymerase chain reaction (PCR) test. The number of polymorphonuclear leukocytes (PMNL) was detected by microscopy.

Results Urethritis was confirmed in only 72/822 (8.76\%) patients by detecting a significant number of PMNL in sediment of FVU, and etiologic diagnosis was established in 68/72 men with diagnosed urethritis. Chlamydia trachomatis was detected as the most common cause of urethritis (47.22\%), followed by Mycoplasma genitalium (12.50\%) and Neisseria gonorrhoeae $(9.72 \%)$. In patients with nongonococcal urethritis (NGU), M. genitalium was found as the second most common 
NGU pathogen. Microbiological analysis was required the most frequently for screening and control purposes (340/822, $41.36 \%)$, and in patients with chronic prostatitis (105/822, $12.77 \%$ of all patients). ST pathogens were detected more frequently in patients with urethritis than in patients from other diagnostic groups $(\mathrm{p}<0.01)$, while Ureaplasma urealyticum was detected without statistically significant difference among different diagnostic groups of patients $(p>0.05)$.

Conclusion Urethritis was confirmed in less than $10 \%$ of patients, and the most samples collected in routine of clinical praxis are from patients without urethritis. In patients with urethritis multiplex PCR test can detect etiology quickly and reliable in almost all cases from noninvasive sample.

Disclosure No significant relationships.

\section{P055 LONDON SEXUAL HEALTH PROGRAMME - DEVELOPING INNOVATIVE SOLUTIONS TO OPEN ACCESS SEXUAL HEALTH SERVICES} ${ }^{4}$ Ryan Kinsella*. ${ }^{1}$ City of London Corporation, Sexual Health Team, London, UK; ${ }^{2}$ City of London Corporation, London, UK; ${ }^{3}$ Greater Glasgow and Clyde NHS, Sandyford Initiative, Glasgow, UK; ${ }^{4}$ Preventx, NH, UK

10.1136/sextrans-2019-sti.259

Background Demand for sexual health services is increasing at a time when public funding has reduced. This Programme is a partnership of local government, working with England's National Health Service to improve access to sexual health services. A key part of our vision was to develop an online sexual health triage, home testing and treatment service, which work with a network of over 40 clinics.

Methods In order to achieve this, the Programme undertook widespread engagement across the city. This included a range of activities with service users, for example, online and clinicbased surveys and focus groups with underrepresented groups. Engagement also took place with clinicians, payorst and other stakeholders. Questions were asked to service users about their usage of services and the acceptability of new innovations, including online based self-sampling options. Clinical organisations were asked about the feasibility of online services being part of the clinical pathway. Alongside this engagement project, new governance arrangements, which took the form of legally binding agreements that enabled the parameters of a pan-London service procurement to take place. At the same time, payors agreed to align pricing for activity in clinics, to support system sustainability for both sides.

Results While many respondents said they value being able to go to a sexual health clinic, over half said they would use an online alternative.. With the governance for this partnership finalised: aligned service specifications for both clinic provision and an e-service,could take place for the online service which launched in January '18. The e-service has registered over $120 \mathrm{k}$ users since then

Conclusion The pressure of growing sexual health need on reduced public resource are self evident, doind more of the same was not a viable or sustainable option. With efficiencies brought about through citywide cooperation, robust clinical and commissioner governance structures, access to services in London has been enhanced.

Disclosure No significant relationships.

\section{P056}

THE ACCEPTABILITY OF DIFFERENT HIV TESTING OPTIONS AMONG YOUNG MEN LIVING IN VANCOUVER, CANADA: A QUALITATIVE STUDY

${ }^{1}$ Caroline Mniszak*, ${ }^{1}$ Anna Carson, ${ }^{1}$ Amy Prangnell, ${ }^{2}$ Jean Shoveller, ${ }^{1}$ Rod Knight. ${ }^{1}$ British Columbia Centre on Substance Use, Vancouver, Canada; ${ }^{2}$ University of British Columbia, School of Population and Public Health, Vancouver, Canada

\subsection{6/sextrans-2019-sti.260}

Background In British Columbia (BC), three testing options are available: nominal, non-nominal and anonymous. Little is known, however, about the factors that influence the acceptability of the different testing strategies, particularly among young men, a group with disproportionately low HIV testing rates.

Methods We draw on data from in-depth, semi-structured interviews with 45 young men (18-30) in Vancouver, BC, in order to identify the factors that influence the acceptability of different HIV testing options.

Results Most participants described not being aware that there were options other than nominal testing available in Vancouver. Upon learning about non-nominal and anonymous testing options, participants described seeing the value of non-nominal testing, insofar as it safeguards their privacy while at the same time providing a pathway to HIV-related health care for those who test positive. Many were concerned, however, that anonymous testing would present challenges to treatment and care for those who test positive. Others expressed concerns about the implications for public health not having access to accurate and up-to-date information about the 'state' of the HIV epidemic. Nevertheless, while participants did not tend to describe anonymous testing as something they would opt for in the future, almost all of the participants felt offering anonymous testing as an option is an important strategy to reduce barriers for key groups of young men (e.g., those living in rural communities).

Conclusion Based on our results, offering non-nominal and anonymous HIV testing represents an important step in increasing the accessibility of HIV testing for some groups of young men who found these approaches both acceptable and preferable (e.g., over nominal testing). However, within our sample, participants were not aware that anonymous and nonnominal testing were available. Developing clear and easy-tounderstand communication strategies about the different approaches to testing may enhance opportunities for uptake of these approaches.

Disclosure No significant relationships.

\section{P057 THE BRAZILIAN STRATEGY FOR HIV SELF-TESTS FREE DISTRIBUTION IN PUBLIC HEALTH}

${ }^{1}$ Mariana Villares, ${ }^{2}$ Pâmela Gaspar*, ${ }^{2}$ Alisson Bigolin, ${ }^{2}$ José Neto, ${ }^{2}$ Regina Comparini, ${ }^{2}$ Adele Benzaken, ${ }^{2}$ Gerson Fernando Pereira. ${ }^{1}$ Ministry of Health of Brazil, Department of Surveillance, Prevention and Control of TISS, HIVIAIDS And Viral Hepatites, Brasilia, Brazil: ${ }^{2}$ Ministry of Health of Brazil, Department of Surveillance, Prevention and Control of Sexually Transmitted Infections, HIVIAIDS and Viral Hepatitis, Brasilia, Brazil

\subsection{6/sextrans-2019-sti.261}

Background In 2017 there were 866 thousand PLHIV in Brazil. Among them, $84 \%$ knew their status. Innovative strategies are fundamental to increase access to testing in a country with a continental dimension and a concentrated epidemic. The $\mathrm{MoH}$ acquired 400 thousand HIVST to reach 